\title{
INVESTIGACIÓN ORIGINAL:
}

\section{ESTUDIO IN-VITRO. SUSCEPTIBILIDAD A LA PIGMENTACIÓN DE VITROCERÁMICAS}

IN-VITRO STUDY. SUSCEPTIBILITY TO THE PIGMENTATION OF VITROCERAMICS

\author{
Milton Rodrigo Andrade Ponce ${ }^{1}$
}

1 Odontólogo. Especialista en Rehabilitación Oral.

Universidad San Francisco de Quito.

milton.andradep@usfq.edu.ec

\author{
Correspondencia: \\ milton.andradep@usfq.edu.ec
}

Recibido: 09-05-2020

Aceptado: 30-07-2021

Publicado: 01-07-2021

https://doi.org/10.53591/eoug.v4i2.1198

\section{RESUMEN}

El presente estudio tiene como fin determinar el rol del tratamiento de superficie en la susceptibilidad a la pigmentación de las vitrocerámicas, al evaluar por medición digital estandarizada y mediante cálculo en base a la fórmula matemática CIE76 $\Delta \mathrm{E}$, muestras cerámicas feldespáticas y a base de disilicato de litio, luego de haber aplicado micro arenado con óxido de aluminio de $50 \mu \mathrm{m}$ y grabado con ácido hidrofluorhídrico al 9,5\% y sumergir las muestras en vino tino y vinagre balsamico. Los resultados, indican que existió diferencia de color estadísticamente significativa en la dimensión b_, apreciable en los diferentes grupos de experimentación, concluyendo que la susceptibilidad a la pigmentación está relacionada al tratamiento de superficie y al agente pigmentante.

Palabras clave: Cerámica, porcelana, pigmentación, Delta E, susceptibilidad, arenado

\section{ABSTRACT}

The present study aims to determine the role of surface treatment in the susceptibility to pigmentation of glass ceramics, evaluating by standardized digital measurement and calculation based on the mathematical formula CIE76 $\Delta \mathrm{E}$, on feldspathic ceramics and lithium disilicate based ceramics samples, after having applied micro sandblasting with aluminum oxide of $50 \mu \mathrm{m}$ and etching with $9.5 \%$ hydrofluoric acid and immersing the samples in red wine and balsamic vinegar. The results indicate that there was a statistically significant color difference in LAB dimension $b_{-}$, which is appreciable in the different experimental groups, concluding that The susceptibility to pigmentation is related to the surface treatment and the tinting agent. Key words: Ceramics, porcelain, pigmentation, Delta E, susceptibility, sandblasting, etching

\section{INTRODUCCIÓN}

Las porcelanas empleadas en odontología presentan propiedades mecánicas y ópticas que las vuelven la mejor opción al momento

de realizar restauraciones biomiméticas, que tengan estabilidad a largo plazo tanto en color y forma [1]. En cuanto a la estabilidad de color, el presente estudio busca correlacionar la resistencia a la pigmentación de las porcelanas odontológicas con tratamientos de superficie, comúnmente usados para protocolos de adhesión [2].

En 2015, Stefano Gracis y su equipo de colaboradores, modifican la clasificación microestructural dividiendo en aquellas con matriz vítrea, policristalinas y agregando las porcelanas de matriz resinosa, que según la ADA son resinas compuestas, pero por poseer propiedades y contenido vítreo, tiene un comportamiento clínico igual al de las cerámicas [3].

En odontología, para unir dos materiales diferentes es necesario aumentar la energía superficial libre; aumentado la energía superficial, aumenta la humectabilidad de la superficie para el agente cementante [4]. Es recomendable realizar tratamientos de la superficie con el objetivo de crear retenciones micro mecánicas y exponer grupos hidroxilos con el fin de promover retención química, la cual es responsable de la estabilidad a largo plazo de la unión entre las cerámicas dentales, el agente cementante resinoso y el sustrato dentario. [5]

Durante los procesos de acondicionamiento de la superficie para optimizar la adhesión, pueden surgir errores humanos, provenientes del clínico o del técnico en prótesis 


\section{REVISTA CIENTÍFICA “ESPECIALIDADES ODONTOLÓGICAS UG”. ISSN: 2600576X ÓRGANO OFICIAL DE LA FACULTAD PILOTO DE ODONTOLOGÍA DE LA UNIVERSIDAD DE GUAYAQUIL}

dental; la literatura describe que los errores de manufactura pueden crear porosidades, burbujas o fisuras donde posibilita, a un pigmento, alojarse. [6]

Los errores por parte del clínico básicamente son al momento de realizar ajustes y acondicionar la restauración para la cementación; si los ajustes oclusales y de adaptación no son correctamente realizados pueden generar fisuras, micro fracturas y porosidades que conducen a un fallo absoluto de la restauración. [7]

El objetivo general de este estudio fue determinar si las acciones clínicas, descritas por la literatura en los protocolos de adhesión, aumentan la susceptibilidad a la pigmentación de las muestras cerámicas, comparando dos tratamientos de superficie y su comportamiento, al sumergir la muestra en diferentes agentes tincionantes por medio de pruebas visuales cualitativas, a través de evaluación visual y cuantitativas, por medio de fotografía estandarizada, utilizando el sistema Cie L*a*b* 1976, y se planteó encontrar relación entre la posible pigmentación y el tratamiento de la superficie en la muestra cerámica y correlacionar los valores DeltaE con los datos del examen visual dicotómico.

\section{MATERIALES Y MÉTODOS}

La metodología aplicada al presente estudio fue de carácter in-Vitro comparativo, cualitativo y cuantitativo donde se aplicó dos tratamientos de superficies en muestras de porcelana feldespática IPS e.max Ceram y vitrocerámica sintética a base de disilicato de litio IPS e.max Press, con dimensiones de $5 \mathrm{~mm} \times 5 \mathrm{~mm}$ y glaseadas con porcelana IPS e.max Ceram - Glaze; posteriormente sumergidas en vino tinto y vinagre balsámico.

Para la agrupación de las muestras se realizó un grupo control, conformado por 28 superficies vitrocerámicas feldespáticas y sintéticas a base de disilicato de litio, sin tratamiento de superficie.

Se destinaron 30 superficies para el grupo Arenado, conformado por las superficies vitrocerámicas feldespáticas y en disilicato de litio, arenadas con óxido de aluminio de $50 \mu \mathrm{m}$ por 15 segundos a 2 bar de presión, $15 \mathrm{~mm}$ de distancia y $75^{\circ}$ con respecto al plano horizontal, en movimientos circulares [8].

Para el grupo grabado HF (grabado de superficie con ácido hidrofluorhídrico al 9,5\%) se destinó 30 superficies vitrocerámicas feldespáticas y sintéticas a base de disilicato de litio tratadas con $\mathrm{HF}$ al 9,5\% durante 120 segundos y 20 segundos [9] respectivamente, lavado posterior al grabado con agua filtrada durante $1 \mathrm{~min}$ [10].

Una vez realizados los tratamientos de superficie se procedió a sumergir las diferentes muestras en vino y vinagre balsámico a $37^{\circ} \mathrm{C}$, durante veinte días calendario, lo que cronológicamente, equivaldría a aproximadamente un año 8 meses de contacto [11]. Se dividió en grupo de experimentación control constituido por 14 muestras sin tratamiento de superficie; con 56 superficies, divididas en 2 subgrupos; 14 FC (feldespato control) y 14 DC (disilicato control) sumergidas en vino tinto y 14 FC y 14 DC sumergidas en vinagre balsámico, por 20 días a temperatura ambiente.

El segundo grupo de experimentación constó de 15 muestras, de cada variable de superficie; 15-FA (feldespato arenado), 15-DA (disilicato arenado), 15-FG (feldespato grabado con HF 9,5\%), 15-DG (disilicato grabado al 9,5\%), es decir 60 superficies en total; sumergidas en vino por 20 días a temperatura ambiente.

El tercer grupo de experimentación constó de 15 muestras de cada variable de superficie 15- FA, 15-DA, 15-FG, 15-DG, es decir 60 superficies en total; estas fueron sumergidas en vinagre balsámico por 20 días a temperatura ambiente.

Para el registro de los datos visuales se tomó fotografías con cámara Réflex Canon Rebel T5 (Canon, Japón), lente macro 100 mm (Canon, Japón), Twin Flash Canon MT-24EX (Canon, Japón) configuración en manual, balance de blancos personalizado con tarjeta gris de 60 puntos del Color Chart (X Rita, Grand Rapids, Michigan), Perfil DNG calibrados con la tarjeta de Color Xrite; velocidad del obturador $1 / 125$, apertura del diafragma F16, sensibilidad del sensor ISO 100, potencia del Flash 1/1, filtro polarizado - Polar Eyes, distancia de enfoque $30 \mathrm{~mm}$. Las mediciones se realizaron al inicio y al final de la prueba, es decir antes y después de la inmersión en los agentes pigmentantes. Por medio de revelado digital en Lightroom 5 , se procedió a medir los valores en el sistema de color de la Comisión Internacional del Color, CIE L.a.b. 1976 en el programa ColorThink Pro 3.0.3, luego estas muestras fueron cepilladas en ambos lados con un cepillo dental con dentífrico por 10 segundos cada superficie [12].

Una vez captadas las muestras visuales por medio del registro fotográfico, los valores fueron obtenidos con el software ColorThink Pro 3.0.3 y la fotografía polarizada con estandarización en la exposición en base al color gris de 60 puntos de la tarjeta XRITE. EL valor $\Delta \mathrm{E}$ se encontró calculando los datos en el mismo software ColorThink Pro 3.0.3 por medio de una calculadora incorporada. La fórmula del $\Delta \mathrm{E}$ : es un procedimiento que figura la distancia entre dos colores en la escala L.A.B; mediante la raíz de la suma de la diferencia del cuadrado de la diferencia de los valores iniciales y finales [13].

$$
\Delta E=\sqrt[2]{\left(L_{2}-L_{1}\right)^{2}+\left(a_{2}-a_{1}\right)^{2}+\left(b_{2}-b_{1}\right)^{2}}
$$

Se comparó los colores medidos iniciales y finales de cada superficies cerámica por medio de la formula $\Delta \mathrm{E}$, para así 


\section{REVISTA CIENTÍFICA “ESPECIALIDADES ODONTOLÓGICAS UG”. ISSN: 2600576X ÓRGANO OFICIAL DE LA FACULTAD PILOTO DE ODONTOLOGÍA DE LA UNIVERSIDAD DE GUAYAQUIL}

determinar si hubo cambios luego de ser sumergidos en vino y vinagre balsámico. En cuanto a la literatura especifica de los estudios de percepción visual, que emplean la fórmula del CIE L.a.b. $1976-\Delta \mathrm{E}$, indican que valores de $\Delta \mathrm{E}$ entre 1.1 a 5.5 se consideran dentro del rango perceptible para el ojo humano [14], si los valores $\Delta \mathrm{E}$ son inferiores a 1.1 es posible que el cambio de color no sea percibido [15]. Al comparar este rango de valores $\Delta \mathrm{E}$, con los estudios de percepción se establece una relación entre valores $\Delta \mathrm{E}$ más bajos y la percepción de un cambio de color, por observadores con más entrenamiento; mientras que para observadores comunes permite un rango $\Delta \mathrm{E}$ más elevado [16].

\section{RESULTADOS}

Los valores obtenidos por medio de la fórmula $\Delta \mathrm{E}$ en un rango del 0 al 10 , fueron los siguientes: (Tabla 1 )

\begin{tabular}{|c|c|c|c|c|c|c|}
\hline Prueba - Vino & FA & DA & DG & FG & CF & CD \\
\hline V1 & 3,2 & 1,0 & 0,0 & 1,4 & 1,4 \\
\hline V2 & 1,4 & 1,0 & 1,4 & 1,0 & 2,0 & 1,0 \\
\hline V3 & 1,0 & 0,0 & 1,0 & 1,0 & 1,0 & 0,0 \\
\hline V4 & 2,2 & 1,4 & 1,0 & 1,0 & 2,0 & 1,0 \\
\hline V5 & 3,2 & 1,4 & 0,0 & 1,0 & 0,0 & 2,0 \\
\hline V6 & 2,2 & 1,0 & 1,0 & 2,2 & 2,0 & 1,0 \\
\hline V7 & 4,6 & 3,2 & 3,2 & 4,1 & 2,2 & 2,0 \\
\hline V8 & 2,0 & 1,4 & 1,4 & 2,2 & 2,0 & 1,4 \\
\hline V9 & 5,4 & 7,1 & 10,3 & 5,0 & 1,0 & 4,0 \\
\hline V10 & 8,2 & 4,1 & 3,2 & 4,1 & 2,0 & 2,0 \\
\hline V11 & 1,4 & 0,0 & 1,0 & 1,0 & 0,0 & 1,0 \\
\hline V12 & 1,4 & 2,0 & 1,0 & 1,0 & 1,0 & 2,0 \\
\hline V13 & 1,0 & 0,0 & 0,0 & 1,4 & 0,0 & 0,0 \\
\hline V14 & 1,0 & 0,0 & 0,0 & 1,4 & 0,0 & 1,4 \\
\hline V15 & 1,4 & 1,4 & 0,0 & 2,0 & 1,0 \\
\hline
\end{tabular}

Tabla 1 Valores DeltaE, grupo Vino en los grupos de estudio

\begin{tabular}{|c|c|c|c|c|c|c|}
\hline $\begin{array}{c}\text { Prueba - Vinagre } \\
\text { B. }\end{array}$ & FA & DA & DG & FG & CF & $C D$ \\
\hline VB1 & 4,0 & 2,2 & 2,0 & 1,4 & 1,0 & 2,0 \\
\hline VB2 & 4,0 & 1,0 & 2,0 & 3,2 & 1,4 & 1,4 \\
\hline VB3 & 2,0 & 4,1 & 1,0 & 1,4 & 1,0 & 1,0 \\
\hline VB4 & 3,2 & 2,8 & 1,0 & 2,2 & 0,0 & 1,0 \\
\hline VB5 & 1,0 & 6,3 & 1,4 & 2,8 & 1,4 & 3,6 \\
\hline VB6 & 1,4 & 5,4 & 1,0 & 4,5 & 1,0 & 1,0 \\
\hline VB7 & 3,2 & 4,1 & 1,4 & 2,2 & 2,2 & 1,4 \\
\hline VB8 & 1,0 & 5,1 & 3,2 & 4,1 & 2,2 & 1,0 \\
\hline VB9 & 1,4 & 8,2 & 4,6 & 3,6 & 1,4 & 2,2 \\
\hline VB10 & 2,2 & 2,2 & 3,6 & 3,2 & 1,4 & 0,0 \\
\hline VB11 & 3,0 & 1,4 & 1,4 & 1,4 & 1,0 & 1,4 \\
\hline VB12 & 2,0 & 1,0 & 2,0 & 1,4 & 1,0 & 2,2 \\
\hline VB13 & 3,2 & 5,4 & 1,4 & 2,2 & 1,0 & 1,4 \\
\hline VB14 & 3,0 & 1,4 & 1,0 & 2,0 & 1,4 & 2,2 \\
\hline VB15 & 2,2 & 3,0 & 1,0 & 1,4 & 1,0 & 2,0 \\
\hline
\end{tabular}

Tabla 2 Valores DeltaE, grupo Vinagre balsámico en los grupos de estudio.

Para realizar el análisis estadístico de la presente investigación, en base a los resultados obtenidos en las pruebas de laboratorio, es importante señalar que se utilizó los medios tecnológicos disponibles como el Excel 2016 y el software estadístico SPSS v.23 de 


\section{REVISTA CIENTÍFICA “ESPECIALIDADES ODONTOLÓGICAS UG”. ISSN: 2600576X ÓRGANO OFICIAL DE LA FACULTAD PILOTO DE ODONTOLOGÍA DE LA UNIVERSIDAD DE GUAYAQUIL}

IBM, con estas herramientas ha sido posible dar a los resultados un procesamiento estadístico descriptivo mediante tablas y gráficos e inferencial con la aplicación de los estadígrafos que permiten establecer las existencia de significancia estadística en las diferentes relaciones de las variables analizadas.

En los resultados de las medias aritméticas, producto de la comparación en pares por el tipo de material y tipo de compuesto de inmersión (vino o vinagre), se observa que existen diferencias en cada uno de los pares contrastados, por lo cual se establece la prueba estadística para establecer si es significativa y se determina que no presentan diferencias estadísticamente significativas con respecto al grupo control (Gráfico 1).

\section{Gráfico 1 Resultados medias emparejadas por tipo de porcelana de acuerdo al $\Delta E$}

Referente a los valores cuantitativos, en cuanto a los valores b_ de la dimensión de color todos los datos fueron estadísticamente significativos al sr p $>0,05$, por lo que podemos determinar que si existió cambio de color significativo en los tonos rojo azulados.

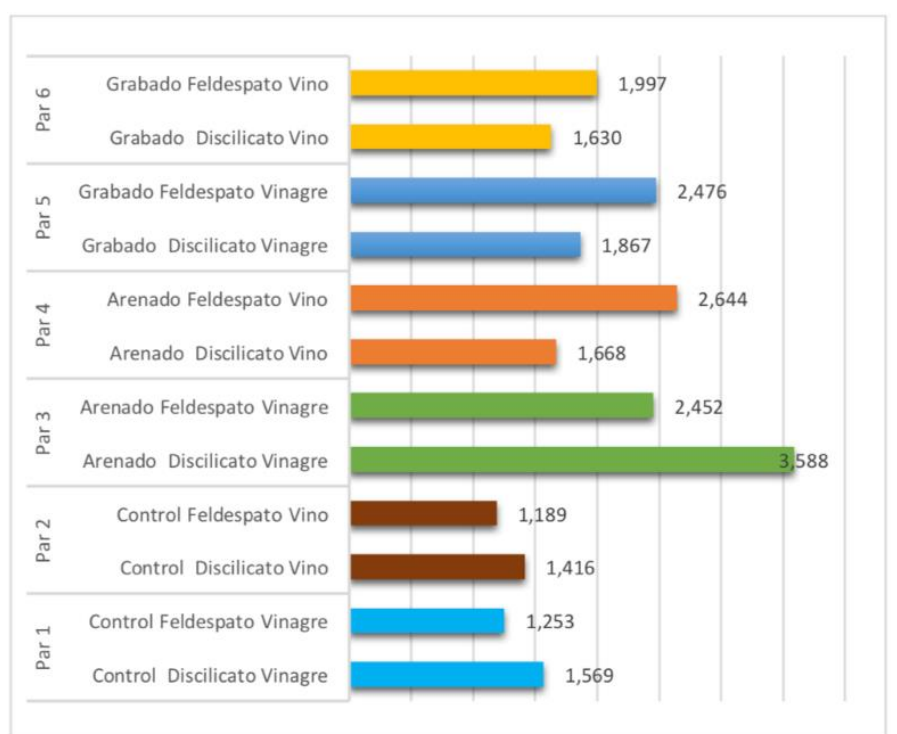

La prueba dicotómica para determinar los valores cualitativos, se realizó en 5 observadores de diferente sexo y nivel de entrenamiento visual, se usó una fotografía estandarizada inicial y una final de cada grupo y se solicitó a los observadores usar los parámetros de SI o No en cuanto a la detección de un cambio de color (Tabla 3, Tabla 4). Los resultados fueron los siguientes:

\begin{tabular}{|c|c|c|c|c|c|c|c|c|c|c|c|c|}
\hline \multirow[t]{2}{*}{$P^{\prime}$} & \multicolumn{2}{|c|}{$\mathrm{FA}$} & \multicolumn{2}{|c|}{ DA } & \multicolumn{2}{|c|}{ DG } & \multicolumn{2}{|c|}{ FG } & \multicolumn{2}{|c|}{ FC } & \multicolumn{2}{|c|}{$D C$} \\
\hline & $\Delta \mathrm{E}$ & E. V & $\Delta \mathrm{E}$ & E. V & $\Delta \mathrm{E}$ & E. V & $\Delta \mathrm{E}$ & E. V & $\Delta \mathrm{E}$ & E. V & $\Delta \mathrm{E}$ & E. $V$ \\
\hline VB1 & 4,00 & NO & 2,24 & SI & 2,00 & SI & 1,41 & NO & 1,00 & NO & 2,00 & SI \\
\hline VB2 & 4,00 & NO & 1,00 & SI & 2,00 & SI & 3,16 & SI & 1,41 & NO & 1,41 & SI \\
\hline VB3 & 2,00 & NO & 4,12 & SI & 1,00 & SI & 1,41 & NO & 1,00 & SI & 1,00 & NO \\
\hline VB4 & 3,16 & SI & 2,83 & SI & 1,00 & NO & 2,24 & NO & - & NO & 1,00 & SI \\
\hline VB5 & 1,00 & NO & 6,32 & SI & 1,41 & NO & 2,83 & SI & 1,41 & NO & 3,61 & SI \\
\hline VB6 & 1,41 & SI & 5,39 & SI & 1,00 & SI & 4,47 & SI & 1,00 & NO & 1,00 & SI \\
\hline VB7 & 3,16 & NO & 4,12 & SI & 1,41 & SI & 2,24 & NO & 2,24 & NO & 1,41 & NO \\
\hline VB8 & 1,00 & NO & 5,10 & SI & 3,16 & NO & 4,12 & SI & 2,24 & NO & 1,00 & SI \\
\hline VB9 & 1,41 & NO & 8,25 & SI & 4,58 & SI & 3,61 & SI & 1,41 & NO & 2,24 & SI \\
\hline VB10 & 2,24 & SI & 2,24 & SI & 3,61 & No & 3,16 & SI & 1,41 & SI & - & NO \\
\hline VB11 & 3,00 & SI & 1,41 & SI & 1,41 & NO & 1,41 & NO & 1,00 & SI & 1,41 & SI \\
\hline VB12 & 2,00 & SI & 1,00 & SI & 2,00 & NO & 1,41 & NO & 1,00 & SI & 2,24 & SI \\
\hline VB13 & 3,16 & NO & 5,39 & NO & 1,41 & NO & 2,24 & SI & 1,00 & NO & 1,41 & NO \\
\hline VB14 & 3,00 & SI & 1,41 & NO & 1,00 & NO & 2,00 & NO & 1,41 & SI & 2,24 & NO \\
\hline VB15 & 2,24 & SI & 3,00 & NO & 1,00 & NO & 1,41 & SI & & & & \\
\hline Media & 2,45 & NO & 3,59 & SI & 1,87 & NO & 2,48 & $\mathrm{SI}$ & 1,17 & NO & 1,57 & SI \\
\hline
\end{tabular}

Tabla 3 Valores dicotómicos que concuerdan con los índices de percepción visual $\Delta$ E para grupos sumergidos en vinagre balsámico 
REVISTA CIENTÍFICA “ESPECIALIDADES ODONTOLÓGICAS UG”. ISSN: 2600576X ÓRGANO OFICIAL DE LA FACULTAD PILOTO DE ODONTOLOGÍA DE LA UNIVERSIDAD DE GUAYAQUIL

\begin{tabular}{|c|c|c|c|c|c|c|c|c|c|c|c|c|}
\hline \multicolumn{2}{|c|}{ Vino } & \multicolumn{2}{|c|}{$\mathrm{FA}$} & \multicolumn{2}{|c|}{ DA } & \multicolumn{2}{|l|}{ DG } & FG & \multicolumn{2}{|c|}{ FC } & \multicolumn{2}{|c|}{ DC } \\
\hline & $\Delta \mathrm{E}$ & E. V & $\Delta \mathrm{E}$ & E.V & $\Delta \mathrm{E}$ & E. V & $\Delta \mathrm{E}$ & E. V & $\Delta \mathrm{E}$ & E. V & $\Delta \mathrm{E}$ & E. V \\
\hline V1 & 3,16 & SI & 1,00 & NO & - & NO & 1,41 & NO & 1,41 & NO & 1,00 & NO \\
\hline V2 & 1,41 & SI & 1,00 & NO & 1,41 & NO & 1,00 & NO & 2,00 & NO & 1,00 & NO \\
\hline V3 & 1,00 & NO & - & NO & 1,00 & SI & 1,00 & NO & 1,00 & NO & - & NO \\
\hline V4 & 2,24 & SI & 1,41 & NO & 1,00 & NO & 1,00 & NO & 2,00 & SI & 1,00 & NO \\
\hline V5 & 3,16 & SI & 1,41 & NO & - & NO & 1,00 & NO & - & NO & 2,00 & SI \\
\hline V6 & 2,24 & NO & 1,00 & SI & 1,00 & NO & 2,24 & SI & 2,00 & NO & 1,00 & SI \\
\hline V7 & 4,58 & NO & 3,16 & SI & 3,16 & SI & 4,12 & SI & 2,24 & SI & 2,00 & SI \\
\hline V8 & 2,00 & SI & 1,41 & SI & 1,41 & NO & 2,24 & NO & 2,00 & NO & 1,41 & SI \\
\hline V9 & 5,39 & SI & 7,07 & NO & 10,3 & $\mathrm{SI}$ & 5,00 & NO & 1,00 & No & 4,00 & NO \\
\hline V10 & 8,25 & SI & 4,12 & SI & 3,16 & $\mathrm{SI}$ & 4,12 & NO & 2,00 & NO & 2,00 & NO \\
\hline V11 & 1,41 & No & - & NO & 1,00 & NO & 1,00 & NO & - & NO & 1,00 & NO \\
\hline V12 & 1,41 & NO & 2,00 & NO & 1,00 & NO & 1,00 & NO & 1,00 & NO & 2,00 & NO \\
\hline V13 & 1,00 & NO & - & NO & - & NO & 1,41 & NO & - & NO & - & NO \\
\hline V14 & 1,00 & NO & - & NO & - & NO & 1,41 & NO & - & NO & 1,41 & NO \\
\hline V15 & 1,41 & SI & 1,41 & NO & - & NO & 2,00 & NO & & & & \\
\hline Media & 2,64 & SI & 1,67 & NO & 1,63 & NO & 2,00 & NO & 1,11 & NO & 1,42 & NO \\
\hline
\end{tabular}

Tabla 4 Valores dicotómicos que concuerdan con los índices de percepción visual $\Delta$ E para grupos sumergidos en vino

Analizando los valores de la evaluación visual y al correlacionar las respuestas de los observadores con los valores $\Delta$ E, se pudo apreciar si realmente estos índices de percepción visual son confiables. Con un total de 880 evaluaciones realizadas entre los 5 observadores, se obtuvo 598 respuestas correlacionadas con los valores $\Delta \mathrm{E}$, lo que equivale a un $68 \%$ de exactitud, lo cual determina que existe correlación, pero aun así, la prueba visual sigue siendo un examen subjetivo y dependerá siempre de los observadores y el nivel de entrenamiento visual de cada uno.

Analizando los valores L.a.b. individualmente, podemos identificar principalmente diferencias estadísticamente significativas para el grupo L.a.b._b (Tabla 5) con respecto a los valores a y L, lo que indica una variación del color en el rango de amarillo, mezclado con el tono rojizo del grupo a; forma un color secundario; según los valores obtenidos las muestras modificaron su color de blanco a marrón.

\begin{tabular}{|c|c|c|c|c|c|c|c|c|}
\hline & \multicolumn{2}{|c|}{ Diferencias emparejadas } & \multirow{3}{*}{$\begin{array}{l}\text { Media de error } \\
\text { octándar }\end{array}$} & & & \multirow[t]{3}{*}{$\mathrm{t}$} & \multirow[t]{3}{*}{ gl } & \multirow{3}{*}{$\begin{array}{c}\text { p-valor } \\
\text { Sig (bilateral) }\end{array}$} \\
\hline & Media & Desviación estándar & & \multicolumn{2}{|c|}{$\begin{array}{l}95 \% \text { de intervalo de confianza de la } \\
\text { diforancia }\end{array}$} & & & \\
\hline & & & & Inferior & Superior & & & \\
\hline CV - GVB Final Lab_L & -1840 & 26277 & 49659 & -1203 & 83487 & -371 & 27 & 714 \\
\hline CV - GVB Final Lab_a & 14565 & 485498 & 091751 & -0426 & 33390 & 1587 & 27 & 124 \\
\hline CV - GVB Final Lab_b & -4455 & 770969 & 1457 & .7444 & -14650 & -306 & 27 & 5 \\
\hline CVB - AVB Final Lab_L & -269 & 25286 & 4779 & -1249 & 07119 & -056 & 27 & 579 \\
\hline CVB - AVB Final Lab_a & 01551 & 043428 & 00821 & -00133 & 03235 & 189 & 27 & 70 \\
\hline CVB - AVB Final Lab_b & -326 & 08020 & 01516 & -06373 & -00153 & -215 & 27 & 40 \\
\hline CV Final - GV Final Lab_L & 21479 & 3384 & 063951 & -10974 & 1527 & 336 & 27 & 740 \\
\hline CV Final - GV Final Lab_a & 06069 & 4834 & 00913 & -01267 & 02481 & 664 & 27 & 512 \\
\hline CV Final - GV Final Lab_b & -10107 & 9818 & 18554 & -13914 & -63005 & -5448 & 27 & 0 \\
\hline CV - AV Final Lab_L & 63895 & 40284 & 7613 & -9231 & 2201 & 839 & 27 & 409 \\
\hline CV - AV Final Lab_a & -0437 & 81618 & 1542 & -36015 & 273 & -283 & 27 & 779 \\
\hline CV - AV Final Lab_b & -8361 & 4972 & 09396 & -10289 & -06433 & -8898 & 27 & 0 \\
\hline GV Inicial - Final Lab_L & -21121 & 17131 & 312762 & -8509 & 42846 & -675 & 29 & 505 \\
\hline GV Inicial - Final Lab_a & -1337 & 39944 & 07293 & -2829 & 0154 & -1834 & 29 & 77 \\
\hline GV Inicial - Final Lab_b & -9825 & 9906 & 1809 & -1353 & -61279 & -5433 & 29 & 0 \\
\hline AV Inicial - Final Lab_L & 10549 & 2541 & 46392 & 1061 & 20037 & 2274 & 29 & 31 \\
\hline AV Inicial - Final Lab_a & -23867 & 6466 & 1180 & -4801 & 00275 & -2022 & 29 & 52 \\
\hline AV Inicial - Final Lab_b & -85299 & 50956 & 09303 & -10433 & -6627 & -9169 & 29 & 0 \\
\hline CV Inicial - Final Lab_L & -0668 & 15714 & 29696 & -676 & 54251 & -225 & 27 & 824 \\
\hline CV Inicial - Final Lab_a & -2370 & 39749 & 07512 & -3912 & -0829 & -3155 & 27 & 4 \\
\hline CV Inicial - Final Lab_b & .08705 & 5037 & 09520 & -2824 & 10829 & -914 & 27 & 369 \\
\hline GVB Inicial - Final Lab_L & 4063 & 1967 & 3591 & -3282 & 11407 & 1131 & 29 & 267 \\
\hline GVB Inicial - Final Lab_a & 00286 & 3368 & 06149 & -1229 & 1286 & 47 & 29 & 963 \\
\hline GVB Inicial - Final Lab_b & -1249 & 5222 & 0953 & -1444 & -1054 & -13100 & 29 & 0 \\
\hline AVB Inicial - Final Lab_L & 6197 & 31697 & 5787 & -5638 & 1803 & 1071 & 29 & 293 \\
\hline AVB Inicial - Final Lab_a & 0184 & 3253 & 0594 & -1031 & 1398 & 309 & 29 & 759 \\
\hline AVB Inicial - Final Lab_b & -1225 & 6998 & 1278 & -1486 & -9634 & -9586 & 29 & 0 \\
\hline AVB Inicial - Final Lab_L & 6197 & 31697 & 5787 & -5638 & 1803 & 1071 & 29 & 293 \\
\hline AVB Inicial - Final Lab_a & 0184 & 3253 & 0594 & -1031 & 1398 & 309 & 29 & 759 \\
\hline AVB Inicial - Final Lab_b & -1225 & 6998 & 1278 & -1486 & -9634 & -9586 & 29 & 0 \\
\hline AVB Inicial - Final Lab_L & 6197 & 31697 & 5787 & -5638 & 1803 & 1071 & 29 & 293 \\
\hline AVB Inicial - Final Lab_a & 0184 & 3253 & 0594 & -1031 & 1398 & 309 & 29 & 759 \\
\hline AVB Inicial - Final Lab_b & -1225 & 6998 & 1278 & -1486 & -9634 & -9586 & 29 & 0 \\
\hline
\end{tabular}

Tabla 5 Valores de la dimensión de color en valores $L_{-} a_{-} b_{-}$ 


\section{REVISTA CIENTÍFICA “ESPECIALIDADES ODONTOLÓGICAS UG”. ISSN: 2600576X ÓRGANO OFICIAL DE LA FACULTAD PILOTO DE ODONTOLOGÍA DE LA UNIVERSIDAD DE GUAYAQUIL}

\section{DISCUSIÓN}

Los estudios del color y los índices de confiabilidad entre la percepción de color y los valores objetivos de la formula $\Delta \mathrm{E}$, varían dependiendo del observador, de acuerdo a los estudios revisados, se permiten valores $\Delta \mathrm{E}$ entre $1 \mathrm{y} 5$. De acuerdo a la prueba dicotómica realizada en este estudio se puede observar que los valores $\Delta \mathrm{E}$ entre 1 y 2 no fueron percibidos por los diferentes observadores, los valores $\Delta \mathrm{E}$ mayores a 3 fueron percibidos por la mayoría de los observadores. Entre los autores la clasificación de Sascha Hein se presenta de manera objetiva y clara, además de correlacionar los valores descritos con los valores expuestos en el presente estudio.

De acuerdo al tipo de tratamiento aplicado a las superficies, los valores obtenidos $\Delta \mathrm{E}$, que cuantifican el cambio de color entre la muestra inicial y final, son mayores para los grupos tratados con micro arenado de superficie, con una media de 2,16 para el grupo AV y de 3,05 para el grupo AVB, indiferente del tipo de material.

En cuanto al análisis estadístico del tipo de tratamiento aplicado a las muestras, indica diferencias estadísticamente significativas para ambos grupos de inmersión en relación al grupo control, sometidos al micro arenado de superficie; mientras que el grabado $\mathrm{HF}$ de superficie sólo indica diferencias estadísticamente significativas para el grupo de inmersión en vinagre. Según los valores $\Delta \mathrm{E}$ obtenidos, los grupos de inmersión en vinagre balsámico, tratados con grabado HF y micro arenado de superficies, presentan valores $\Delta \mathrm{E}$ más elevados que los grupos de inmersión en Vino, indiferente del tratamiento de superficie; encontrando diferencias significativamente estadísticas. Puede justificarse dichas diferencias a la diferencia de acidez en el pH de ambas sustancias, esto nos indica que existe una influencia entre el tipo de sustancia en la que se realiza la inmersión [17]

Realizando un análisis más específico en cuanto a los valores entre feldespato y disilicato de litio, se obtuvo que con respecto al grupo control, los tratamientos de superficie aumentaron la susceptibilidad a la pigmentación de las porcelanas. Los grupos de Arenado tuvieron diferencias significativas sin importar la sustancia de inmersión o el material cerámico; mientras que en los grupos de grabado ácido, el feldespato tuvo mayor susceptibilidad a la pigmentación, en el grupo arenado el disilicato de litio demostró valores significativamente más altos.

Los resultados de los valores de grabado ácido en el feldespato y disilicato de litio, pueden indicar que las vitrocerámicas feldespáticas, al requerir mayor tiempo de grabado, aumentan la susceptibilidad a la pigmentación [18], pero debemos considerar que el recubrimiento vítreo que se realiza al glasear la superficie, es a base de feldespato, por lo tanto, al aplicar un tiempo de grabado $\mathrm{HF}$ de 20 segundos sobre el disilicato de litio, como lo indica la literatura, no se llega al tiempo necesario para modificar la estructura del glaseado vítreo [19]. Considerando que las muestras se realizaron sobre disilicato de litio IPS e.max Press y feldespato IPS e.max Ceram, se sugiere realizar estudios futuros en materiales IPS e.max CAD debido a la evidencia en cuanto a la diferencia de las propiedades mecánicas. [20]

En contraste con un estudio previo realizado por el Dr. Víctor Rodas (Gráfico 2), el tratamiento de superficie presenta congruencia en cuanto a valores Delta E con otros tratamientos de superficie (pulido mecánico, limpieza con punta de ultrasonido, fresado, en el mismo agente tincionantes; los resultados entre tratamientos de superficie no fueron estadísticamente significativos en relación a los grupos control.

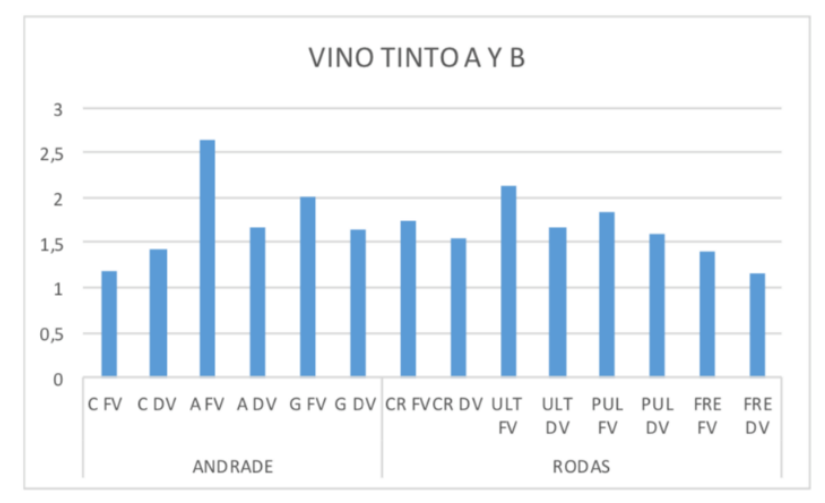

Gráfico 2 Correclación de valores de tratamientos de superfice en estudios posteriores bajo los mismos criterios.

En cuanto a la diferencia entre agentes tincionantes (Gráfico 3), los resultados entre vino tinto y vinagre balsámico, entre los dos estudios; la diferencia de valores fue estadísticamente significativa en comparación con el grupo control y el grupo vinagre balsámico , indicándonos que el cambio de color está directamente relacionado con el agente pigmentante [21]



Gráfico 3 Valores DeltaE comparativos entre vino tinto y vinagre balsámico 


\section{REVISTA CIENTÍFICA “ESPECIALIDADES ODONTOLÓGICAS UG”. ISSN: 2600576X ÓRGANO OFICIAL DE LA FACULTAD PILOTO DE ODONTOLOGÍA DE LA UNIVERSIDAD DE GUAYAQUIL}

\section{CONCLUSIONES}

Considerando las limitaciones del presente estudio, en base a la experimentación y a los resultados obtenidos se puede concluir que:

- $\quad$ El tratamiento de superficie tiene relación directa con la susceptibilidad a la pigmentación.

- $\quad$ El micro arenado aumenta la susceptibilidad a la pigmentación en comparación al grabado HF 9,5\% con un $p$ $=0,013$ para los grupos sumergidos en vinagre balsámico. En cuanto al grupo vino no presento diferencia estadísticamente significativa con un $\mathrm{p}=0,376$.

- $\quad$ Se podrán aceptar valores $\Delta \mathrm{E}$ entre 0,5 a 2,0, como referencia a los límites de tolerancia visual.

\section{REFERENCIAS BIBLIOGRAFICAS}

1. Ambrosi M, Santoni S, Giorgi R, EmilianoFNTPB. High-performance and anti-stain coating for porcelain stoneware tiles based on nanostructured zirconium compounds. Journal of Colloid and Interface Science. 2014;432:117-127.

2. Magne P, Belser U. Restauraciones de Porcelana Adherida en Dientes Anteriores Metodo Biomimético. Vol 1. Barcelona: Quintessence; 2004.

3. Gracis S, Thompson V, Ferencz J, Silva N, Bonfante E. A New Classification System for All- Ceramic and Ceramic-like Restorative Materials. The International Journal of Prosthodontics. 2015;28(3):227-235.

4. Lu Y, Tseng H, Shih Y, Lee S. Effects of surface treatments on bond strength of glass- inßltrated ceramic. Journal of Oral Rehabilitation. 2001 805813.

5. Ramakrishnaiah R, Alkheraif A, Divakar D, Matinlinna J, Vallittu P. The Effect of Hydrofluoric Acid Etching Duration on the Surface Micromorphology, Roughness, and Wettability of Dental Ceramics. International Journal of Molecular Science. 2016;17:822-839.

6. Albakry M, Guazzatoa M, Swain M. Effect of sandblasting, grinding, polishing and glazing on the flexural strength of two pressable all-ceramic dental materials. Journal of Dentistry. 2004;32:91-99.

7. Zhang Y, Sailer I, Lawn B. Fatigue of dental ceramics. Journal of Dentistry. 2014;41:1135- 1147.

8. Rudawska A, Danczak I, Müller M, Valasek P. The effect of sandblasting on surface properties for adhesion. International Journal of Adhesion \& Adhesives. 2016;70:176-190.

9. Puppin J, Sundfeld D, Puppin RM, Costa AR, Correr AB, Borges GA, Sinhoreti MAC, Correr-Sobrinho L. HF concentration and etching times on lithium disilicate glass-ceramic. Dental Materials. 2016;32:e83.
10. Kina S, Bruguera A. Invisible. Restauraciones Estéticas Cerámicas. Sao Paulo : Dental Press Editora; 2008.

11. Alharbi A, Ardu S, Bortolotto T, Krejci I. Stain susceptibility of composite and ceramic CAD/CAM blocks versus direct resin composites with different resinous matrices. Odontology. 2017;105(2):162169.

12. Hein S, Tapia J, Bazos P. eLABor_aid: a new approach to digital shade management. The International Journal Of Esthetic Dentistry. 2017;12(2):186-202.

13. Ramos D, Almeida A, Garcia M, Giannini M, Puppin R, Ferracane J, Coelho M. Effect of Different In Vitro Aging Methods on Color Stability of a Dental ResinBased Composite Using CIELAB and CIEDE2000 Color-Difference Formulas. Journal of Esthetic and Restorative Dentistry. 2015;27(5):322-330.

14. Bravo D, Bustamante E, Daza V. Influencia del tratamiento de superficie en la resistencia traccional de porcelana feldespática reparada con resina compuesta. Revista Clínica de Periodoncia, Implantología y Rehabilitación Oral. 2014;7(3):123127.

15. Ho B, Tsoi J, Liu D, Lung C, Wong HM, Matinlinna J. Effects of sandblasting distance and angles on resin cement bonding to zirconia and titanium. International Journal of Adhesion \& Adhesives. 2015;62:25-31.

16. Khashayar G, Bain P, Salari S, Dozic A, Kleverlaan C, Feilzer A. Perceptibility and acceptability thresholds for colour differences in dentistry. Journal of Dentistry. 2014;42:637- 644.

17. Venturini A, Prochnowb C, Ramboc D, Gundeld A, Valandro L. Effect of Hydrofluoric Acid Concentration on Resin Adhesion to a Feldspathic Ceramic. The Journal of Adhesive Dentistry. 2015;17(4):313-320.

18. Kato $\mathrm{H}$, Matsumura $\mathrm{H}$, Atsuta $\mathrm{M}$. Effect of etching and sandblasting on bond strength to sintered porcelain of unfilled resin. Journal of Oral Rehabilitation. 2000;27:103-110.

19. Liu D, Tsoi J, Pow E, Wong H. Influence of different etching protocols on the reliability of resin bonding to $\mathrm{CAD} / \mathrm{CAM}$ feldspathic porcelain. International Journal of Adhesion \& Adhesive. 2015;62:18-24.

20. Yoshihara K, Nagaoka N, Maruo Y, Nishigawa G, Iried M, Yoshidae Y, Meerbeek BV. Sandblasting may damage the surface of composite CAD-CAM blocks. Dental Materials. 2017;33:e124-e135. 